\title{
Investigation and Analysis of the Information Available for Pregnant and Lactating Women in 308 Antipsychotic Package Inserts
}

\author{
Xiaoru Yang, Jufeng Li, Jieyi Zhou, Xiaofei Feng, Jiawen Huang* \\ Department of Pharmacy, First Affiliated Hospital, Jinan University, Guangzhou, China \\ Email address: \\ eudaemoniay@139.com (Jiawen Huang) \\ ${ }^{*}$ Corresponding author \\ To cite this article: \\ Xiaoru Yang, Jufeng Li, Jieyi Zhou, Xiaofei Feng, Jiawen Huang. Investigation and Analysis of the Information Available for Pregnant and \\ Lactating Women in 308 Antipsychotic Package Inserts. Journal of Gynecology and Obstetrics. Vol. 9, No. 3, 2021, pp. $79-83$. \\ doi: $10.11648 /$ j.jgo.20210903.15
}

Received: June 8, 2021; Accepted: June 18, 2021; Published: June 25, 2021

\begin{abstract}
Background: Package insert is an important tool to provide safe and reasonable guidance for pregnant and lactating women. Methods: The information on pregnant women in 308 package inserts of 38 kinds of antipsychotics on the market was analyzed. Results: 287 (93.18\%) package inserts in the 308 antipsychotics provide drug use guiding information for pregnant women, while $150(48.70 \%)$ of them made it clear that the drug could be used normally, cautiously or forbidden on pregnant women. $137(44.48 \%)$ of them used different expressions. There were $64(20.78 \%)$ instructions that made it clear that the drug could be used normally, cautiously or forbidden on lactation women. $81(26.30 \%)$ instructions are recommended to stop breastfeeding. $142(46.10 \%)$ instructions for breastfeeding women used different expressions. Contradictions were found in $23.53 \%$ drugs for pregnant women and $29.41 \%$ for lactation women. Conclusion: There are some problems with drug information on pregnant women in package inserts, such as inadequate information and inaccurate description. To ensure safer use, the package inserts of medicines should be further improved.
\end{abstract}

Keywords: Package Inserts, Antipsychotics, Pregnant Women, Lactating Women

\section{Introduction}

Pregnant and lactating women are in a special physiological and psychological stage, and the fetus is closely connected with the mother [1]. Drugs may also enter the fetus through the placenta or milk while acting on the mother, causing adverse effects. Therefore, it is particularly important to provide safe and reasonable medication guidance for pregnant and lactating women. In recent years, the prevalence of mental disorders is increasing, and some studies have found that the age of high incidence of mental disorders that most often require antipsychotic drugs coincides with the optimal childbearing age of women [2]. In addition, due to physical discomfort, parenting pressure and so on, pregnant and lactating women are prone to a variety of mental problems, such as sleep disorders, anxiety, depression and so on [3]. Therefore, for women with mental disorders during pregnancy and lactation, the rational selection of antipsychotic drugs is crucial, and drug package inserts provide detailed information about the use of drugs, which plays an important role in drug use guidance.

The purpose of this paper is to investigate and analyze the medication information labeling of pregnant and lactating women through the package inserts of antipsychotic drugs in China, so as to provide reference for rational clinical use of antipsychotic drugs and promote more standard drug instructions.

\section{Materials and Methods}

A total of 308 package inserts of 34 kinds of antipsychotic drugs in China were collected. They were individually read with particular attention on "Regulations for the Specification of Chemical Drugs and Therapeutic Biological Products", and their standards while referencing the "Drug Administration Instructions and Labeling Management Regulations." The 
drug names, manufacturers and medication information of pregnant and lactating women in the instructions were marked one by one, and the statistics and analysis were made.

\section{Results}

A total of 308 package inserts for antipsychotics were analyzed. According to the types of diseases, they were divided into five categories: sedative and hypnotic drugs, antipsychotics, antianxiety drugs, antimanic drugs and antidepressants. The medication information in the instructions for pregnancy was shown in Table 1, and the medication information for lactation was shown in Table 2.

Table 1. Status of information on a antipsychotic use in pregnant women, from 308 package inserts.

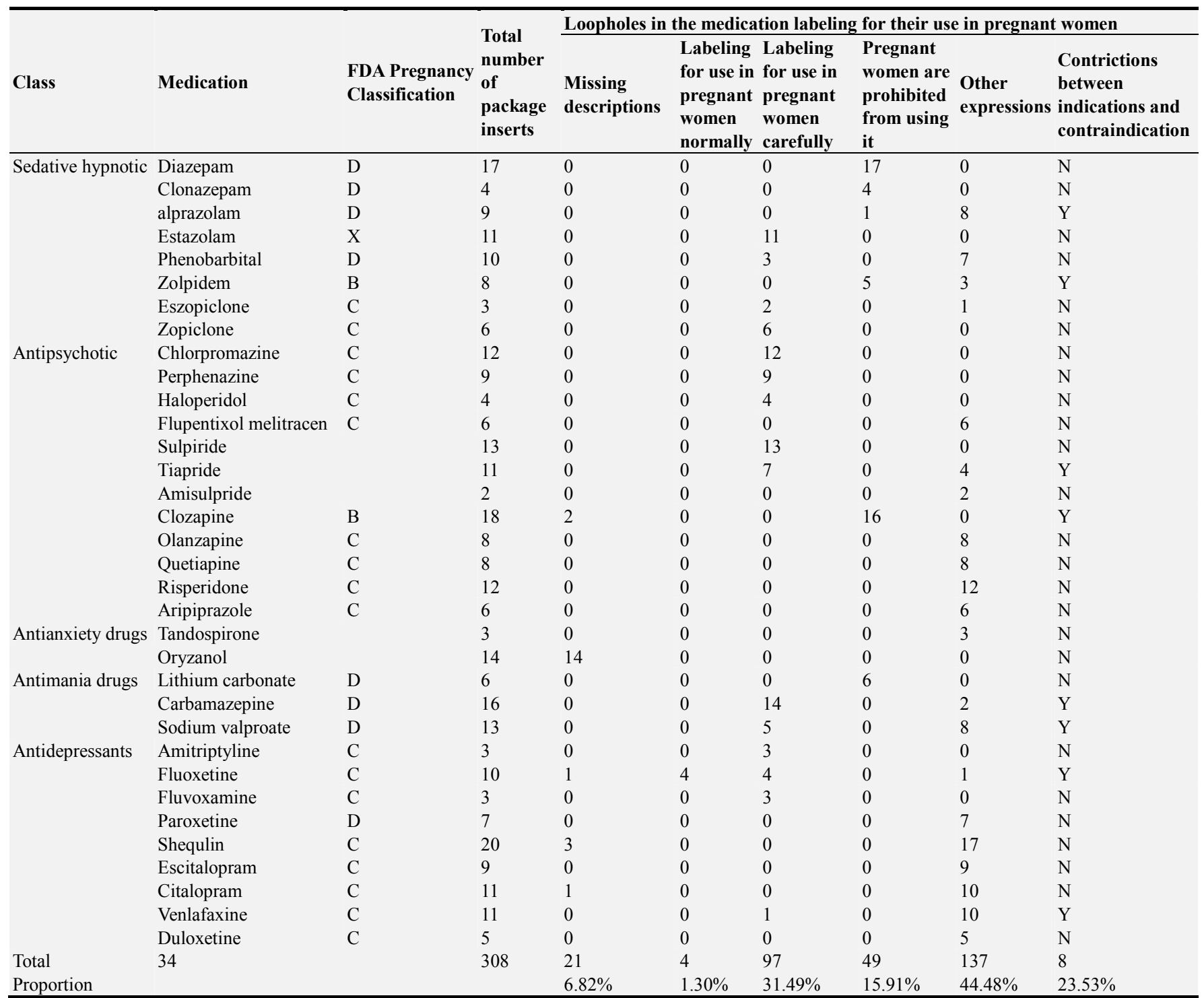

Among the 308 package inserts surveyed in this study, 287 $(93.18 \%)$ listed [medication for pregnant women and lactating women] separately, and marked the relevant information of medication for pregnancy. Among them, 150 cases (48.70\%) clearly marked that the drug could be used normally, used with caution or banned, while 137 cases $(44.48 \%)$ used a variety of tips, including "use only when advantages outweigh disadvantages", "not recommended", "should not be used" and so on. A further $21(6.82 \%)$ of the inserts, including all 14 of the anti-anxiety drug Glovezol, lacked any information regarding pregnancy medication. Among the 38 drugs investigated, 8 kinds $(23.53 \%)$ of different manufacturers had obvious contradictions on medication labeling information for pregnancy, and the profile was coexistence of usable and forbidden drugs.

On the other hand, 287 (93.18\%) listed [medication for pregnant women and lactating women] separately in the package inserts, marking the information related to medication during lactation. Among them, 64 instructions (20.78\%) clearly marked available, cautious or prohibited drugs, 81 instructions $(26.30 \%)$ required lactating women to stop breastfeeding during medication, and 142 instructions (46.10\%) had complex and mixed medication tips for lactating women. Including "weigh the pros and cons", "best not to use", 
"not to use" and so on. In addition, we found that $21(6.82 \%)$ of the instructions lacked any information regarding medication for lactating women, including all 14 of the anti-anxiety drug Glovezol. Among the 38 drugs investigated,
10 kinds (29.41\%) of different manufacturers had obvious contradictions in the labeling information of medication during lactation, with the coexistence of usable and forbidden drugs.

Table 2. Status of information on a antipsychotic use in lactating women, from 308 package inserts.

\begin{tabular}{|c|c|c|c|c|c|c|c|c|c|c|}
\hline \multirow[b]{2}{*}{ Class } & \multirow[b]{2}{*}{ Medication } & \multirow[b]{2}{*}{$\begin{array}{l}\text { Lactation } \\
\text { Classification }\end{array}$} & \multirow[b]{2}{*}{$\begin{array}{l}\text { Total } \\
\text { number } \\
\text { of } \\
\text { package } \\
\text { inserts }\end{array}$} & \multicolumn{7}{|c|}{ Loopholes in the medication labeling for their use in lactating women } \\
\hline & & & & $\begin{array}{l}\text { Missing } \\
\text { descriptions }\end{array}$ & $\begin{array}{l}\text { Labeling } \\
\text { for use in } \\
\text { lactating } \\
\text { women } \\
\text { normally }\end{array}$ & $\begin{array}{l}\text { Labeling } \\
\text { for use in } \\
\text { lactating } \\
\text { women } \\
\text { carefully }\end{array}$ & $\begin{array}{l}\text { Lactating } \\
\text { women are } \\
\text { prohibited } \\
\text { from using } \\
\text { it }\end{array}$ & $\begin{array}{l}\text { Stop } \\
\text { breastfeeding }\end{array}$ & $\begin{array}{l}\text { Other } \\
\text { expressions }\end{array}$ & $\begin{array}{l}\text { Contrictions } \\
\text { between } \\
\text { indications and } \\
\text { contraindication }\end{array}$ \\
\hline \multirow[t]{8}{*}{ Sedative hypnotic } & Diazepam & L3 & 17 & 0 & 0 & 0 & 0 & 0 & 17 & $\mathrm{~N}$ \\
\hline & Clonazepam & L3 & 4 & 0 & 0 & 0 & 4 & 0 & 0 & $\mathrm{~N}$ \\
\hline & alprazolam & L3 & 9 & 0 & 0 & 8 & 1 & 0 & 0 & $\mathrm{Y}$ \\
\hline & Estazolam & L3 & 11 & 0 & 0 & 11 & 0 & 0 & 0 & $\mathrm{~N}$ \\
\hline & Phenobarbital & L4 & 10 & 0 & 0 & 3 & 0 & 0 & 7 & $\mathrm{Y}$ \\
\hline & Zolpidem & L3 & 8 & 0 & 0 & 0 & 4 & 0 & 4 & $\mathrm{Y}$ \\
\hline & Eszopiclone & L3 & 3 & 0 & 0 & 2 & 0 & 0 & 1 & $\mathrm{~N}$ \\
\hline & Zopiclone & L2 & 6 & 0 & 0 & 0 & 0 & 0 & 6 & $\mathrm{~N}$ \\
\hline \multirow[t]{12}{*}{ Antipsychotic } & Chlorpromazine & L3 & 12 & 0 & 0 & 0 & 0 & 12 & 0 & $\mathrm{~N}$ \\
\hline & Perphenazine & L3 & 9 & 0 & 0 & 0 & 0 & 9 & 0 & $\mathrm{~N}$ \\
\hline & Haloperidol & L3 & 4 & 0 & 0 & 0 & 0 & 4 & 0 & $\mathrm{~N}$ \\
\hline & \multicolumn{2}{|c|}{ Flupentixol melitracen } & 6 & 0 & 0 & 0 & 0 & 0 & 6 & $\mathrm{~N}$ \\
\hline & Sulpiride & L3 & 13 & 0 & 0 & 0 & 0 & 13 & 0 & $\mathrm{~N}$ \\
\hline & Tiapride & & 11 & 0 & 0 & 6 & 0 & 1 & 4 & $\mathrm{Y}$ \\
\hline & Amisulpride & & 2 & 0 & 0 & 0 & 2 & 0 & 0 & $\mathrm{~N}$ \\
\hline & Clozapine & L3 & 18 & 2 & 0 & 0 & 0 & 16 & 0 & $\mathrm{~N}$ \\
\hline & Olanzapine & L2 & 8 & 0 & 0 & 0 & 0 & 0 & 8 & $\mathrm{~N}$ \\
\hline & Quetiapine & L2 & 8 & 0 & 0 & 0 & 0 & 0 & 8 & $\mathrm{~N}$ \\
\hline & Risperidone & L2 & 12 & 0 & 0 & 0 & 0 & 0 & 12 & $\mathrm{~N}$ \\
\hline & Aripiprazole & L3 & 6 & 0 & 0 & 0 & 0 & 4 & 2 & $\mathrm{Y}$ \\
\hline \multirow[t]{2}{*}{ Antianxiety drugs } & Tandospirone & & 3 & 0 & 0 & 0 & 0 & 0 & 3 & $\mathrm{~N}$ \\
\hline & Oryzanol & & 14 & 14 & 0 & 0 & 0 & 0 & 0 & $\mathrm{~N}$ \\
\hline \multirow[t]{3}{*}{ Antimania drugs } & Lithium carbonate & L3 & 6 & 0 & 0 & 0 & 0 & 6 & 0 & $\mathrm{~N}$ \\
\hline & Carbamazepine & L2 & 16 & 0 & 0 & 0 & 0 & 0 & 16 & $\mathrm{~N}$ \\
\hline & Sodium valproate & L4 & 13 & 0 & 4 & 5 & 0 & 0 & 4 & $\mathrm{~N}$ \\
\hline \multirow[t]{9}{*}{ Antidepressants } & Amitriptyline & L2 & 3 & 0 & 0 & 1 & 0 & 2 & 0 & $\mathrm{Y}$ \\
\hline & Fluoxetine & L2 & 10 & 1 & 0 & 3 & 0 & 4 & 2 & $\mathrm{Y}$ \\
\hline & Fluvoxamine & L2 & 3 & 0 & 0 & 0 & 2 & 1 & 0 & $\mathrm{~N}$ \\
\hline & Paroxetine & L2 & 7 & 0 & 0 & 2 & 0 & 0 & 5 & $\mathrm{~N}$ \\
\hline & Shequlin & L2 & 20 & 3 & 0 & 0 & 0 & 0 & 17 & $\mathrm{~N}$ \\
\hline & Escitalopram & & 9 & 0 & 0 & 0 & 0 & 9 & 0 & $\mathrm{~N}$ \\
\hline & Citalopram & L2 & 11 & 1 & 0 & 4 & 0 & 0 & 6 & $\mathrm{Y}$ \\
\hline & Venlafaxine & L2 & 11 & 0 & 0 & 1 & 0 & 0 & 10 & $\mathrm{Y}$ \\
\hline & Duloxetine & L3 & 5 & 0 & 0 & 1 & 0 & 0 & 4 & $\mathrm{Y}$ \\
\hline Total & 34 & & 308 & 21 & 4 & 47 & 13 & 81 & 142 & 10 \\
\hline Proportion & & & & $6.82 \%$ & $1.30 \%$ & $15.26 \%$ & $4.22 \%$ & $26.30 \%$ & $46.10 \%$ & $29.41 \%$ \\
\hline
\end{tabular}

\section{Discussion}

Antipsychotics mainly act on the central nervous system and need to pass through the blood-brain barrier to enter the brain tissue for therapeutic effect. Therefore, drugs are fat-soluble, and the larger they are, the faster they will work. When the drug is highly fat soluble, it is also easy to penetrate the placental barrier and enter the fetus, causing adverse effects on the fetus. Therefore, the FDA pregnancy classification of antipsychotics is mostly grade $\mathrm{C}$ and $\mathrm{D}$, and it should be used with caution during pregnancy. Drugs with high fat soluble content are also relatively high in breast milk, which are easy to accumulate in infants' bodies and cause some harm to them. Therefore, the classification of antipsychotics during lactation is mostly L2 and L3, so it is suggested to balance the advantages and disadvantages.

However, this study found that different manufacturers of the same drug during pregnancy and lactation have different indications, and there is even a contradiction between the coexistence of availability and prohibition. This was the case for eight of the 34 drugs studied. In addition, the pregnancy medication instructions of some drugs were inconsistent with the FDA pregnancy safety classification of the drug. For example, most of the instructions of zolpidem tartrate tablets with the FDA pregnancy classification of B suggested "forbidden for pregnant women". Fluoxetine, on the other hand, has a grade $\mathrm{C}$ pregnancy grade, but some instructions suggest "use during pregnancy." In addition, the pregnancy grade of estazolam is X, indicating that the research on animal 
and human drugs and the experience of human drug use indicate that the drug is harmful to the fetus, and it is not beneficial for pregnant women to use the drug, so it should be prohibited for pregnant patients. However, in the 11 instructions investigated, it is noted that pregnant women should use it with caution. Valproate, which is rated L4 during lactation, indicates that there is clear evidence of harm to infants from the use of the drug by lactating women, but that the benefits outweigh the risks. However, in the investigation of the 13 sodium valproate instructions, 4 of them indicated that lactating women can use it properly, while 5 indicated that lactating women should use it with caution.

These conditions can make it problematic for clinical reference to determine whether the drug is usable or not. Studies in recent years have also found problems such as inconsistency and lag in updating medication information for pregnancy in drug instructions [4-6]. Most of the western drugs listed in China are generic drugs. The 12th Five-Year Plan for National Drug Safety requires generic drugs to be consistent with the quality and efficacy of the original drugs, but it does not force the manufacturers that have passed the consistency evaluation to revise the drug instructions. In addition, even if the relevant drug administration departments require the manufacturers to amend the instructions, they do not follow up the revision, and there is also a lack of means to supervise the implementation of the revision of drug instructions and the relevant reward and punishment mechanism [7]. Therefore, there are still inconsistent non-standard phenomena of the same drug label information produced by different manufacturers.

Depression during pregnancy or after childbirth is one of the most common complications of pregnancy and postpartum. More than $14 \%$ of women will suffer from depression during pregnancy or during breastfeeding after childbirth, and the prevalence is increasing year by year [8-10]. Antidepressants are helpful for the treatment of depression during pregnancy, and there is sufficient evidence to show greater benefits for women and fetuses using antidepressants compared to untreated women and fetuses exposed to depressive symptoms [11]. Selective serotonin reuptake inhibitors (SSRIs) are commonly used antidepressant drugs for depression during pregnancy and lactation. They can selectively inhibit serotonin transporters and antagonize the reuptake of serotonin in the presynaptic membrane, leading to the increase of serotonin levels in the synaptic cleft, and thus exert an antidepressant effect.

Most SSRIs are rated by the FDA as Grade $\mathrm{C}$ for pregnancy safety, and may be teratogenic when used in the first trimester or risk of persistent pulmonary hypertension in the newborn when used in the third trimester. Therefore, antidepressants such as SSRIs are recommended for women with depression during pregnancy only when the benefits outweigh the risks. Sertraline, citalopram and escitalopram are recommended as priority when using SSRIs in pregnant women, as these three drugs have higher safety during pregnancy [12]. In addition, sertraline has the lowest risk of neonatal persistent pulmonary hypertension among other SSRIs used in the third trimester, which should be selected as a priority [13]. In a cohort study of prenatal antidepressant exposure, 2327 of 18,487 pregnant women with SSRI exposure were associated with an increased risk of fetal cardiac malformation after adjusting for confounding factors such as maternal age, comorbiditions, and use of other medications (adjusted for $\mathrm{OR}=1.45,95 \% \mathrm{CI}$ : $1.12 \sim 1.88$ ) and atrioventricular septal defect (adjusted $\mathrm{OR}=1.39$, 95\%CI: 1.00 1.93) [14]. Paxil is therefore associated with a greater risk of fetal heart abnormalities than other SSRIs and should be avoided in pregnant women.

For women with depression during lactation, the selection of antidepressants is mainly based on the amount of drugs entering the milk during lactation, for fear of adverse effects on the baby. However, SSRIs will more or less enter the milk. When the benefits of breastfeeding outweigh the disadvantages, the mother can breastfeed; otherwise, breastfeeding is not recommended. Commonly used SSRIs such as paroxetine or sertraline, after taken by lactation women after breastfeeding, is almost undetectable in the baby's blood, which indicates that it is relatively safe for infants and can be preferred. Fluoxetine, with its long clear half-life and cumulative risk, has the highest blood concentration in infants, and breast-feeding is not recommended for women using the drug [15].

\section{Conclusion}

The use of psychotropic substances during pregnancy must consider the effect of the drug on the mother and the fetus, and during lactation the effect of the drug on the infant through milk. Including but not limited to drug addiction, teratogenicity and other risks, in addition to the disease itself or after the use of drugs on maternal mental state and behavior during pregnancy, need to weigh the advantages and disadvantages. When the benefits of drug therapy outweigh the risks, safety drugs are preferred. Drug package inserts provide critical guidance for safe drug use, especially for patients during pregnancy or lactation, a special physical and psychological period. It was found in this survey that in the instructions of antipsychotic drugs currently supplied in China, there are still some non-standard conditions, such as lack of medication labels for pregnant and lactating women and miscellaneous hints. Therefore, it is suggested that the relevant drug administration departments can further refine and improve the relevant laws and regulations on drug instructions, clarify the responsibilities of each department, timely follow up the implementation of the revision of drug instructions of manufacturers and formulate relevant reward and punishment mechanisms, so as to strengthen the standardized management of drug instructions and provide reference for safe and rational drug use in clinical practice.

\section{References}

[1] Wang QM. Investigation and analysis of medication labeling for pregnant and lactating women in 616 drug product inserts [J]. Journal of Practical Medical Techniques, 2016, 23: 667. 
[2] Einarson A. Boskovic R. Use and safety of antipsychotic drugs during pregnancy [J]. Journal of Psychiatric Practice, 2019, 15 (3): 183-192.

[3] Zhai Q. Zhang GF. Liu M. et al. Rational application of antipsychotics in pregnanc [J]. Chinese General Practice, 2019, 22 (30): 3701-3708.

[4] Huang JW. Li JF. Zhang ZD. et al. Analysis of Drug Information on Pregnant Women in 247 Package Inserts of Antiviral Drugs [J]. Pharmacy Today, 2016, 26 (7): 522-524.

[5] Feng N. (2017). Investigation and Analysis of Medication Information on Pregnant Women in 166 Drug Instructions of Anti-diabetic Drugs [J]. China Pharmacy, 2017, 28 (16): 2301-2304.

[6] Niu JR. Li J. Wu S. et a. Analysis of drug use labeling for pregnant and lactating women in 762 drug package inserts [J]. China Pharmacy, 27 (7): 992-994.

[7] Liu YQ. Wang ZJ. Liang YQ. Analysis on the Publication of Revision Notices of Drug Instructions and Related Status in China in 2015-2019 [J]. China Pharmacy, 2019, 30 (21): 2903-2908.

[8] Margulis AV. Kang EM, Hammad TA. Patterns of prescription of antidepressants and antipsychotics across and within pregnancies in a population-based UK cohor [J]. Matern Child Health J. 2014, 18 (7): 1742-1752.

[9] Sabahi A. Sepehri G. Mohsenbeigi M. et al. Patterns of psychotropic medication prescriptions by psychiatrists for private clinic outpatients in kerman province, iran [J]. Sultan Qaboos University Medical Journal. 2014, 14 (3): e382-387.

[10] Hanley GE. Oberlander TF. The effect of perinatal exposures on the infant: antidepressants and depression [J]. Best practice \& research Clinical obstetrics \& gynaecology, 2014, 28 (1): $37-48$.

[11] Muzik M. \& Hamilton SE. Use of antidepressants during pregnancy: what to consider when weighing treatment with antidepressants against untreated depression [J]. Maternal \& Child Health Journal, 2016, 20 (11): 1-12.

[12] Weisskopf E. Fischer CJ. Bickle Graz M. et al. Risk-benefit balance assessment of SSRI antidepressant use during pregnancy and lactation based on best available evidence [J]. Expert opinion on drug safety, 2015, 14 (3): 413-427.

[13] Zhang YY. Li SX. Li ZH. Research progress on the safety of exposure to selective serotonin reuptake inhibitors during pregnancy $[\mathrm{J}]$. Adverse Drug Reactions Journal, 2021, 23 (3): $140-144$.

[14] Bérard A. Zhao JP. Sheehy O. Antidepressant use during pregnancy and the risk of major congenital malformations in a cohort of depressed pregnant women: an updated analysis of the quebec pregnancy cohort [J]. Bmj Open, 2017, 7 (1): $\mathrm{e} 013372$.

[15] Yu DS. Effects of antidepressants on pregnancy and lactation [J]. Journal of Clinical Psychiatry, 2012, 22 (1): 60-62. 